\title{
Bounds on charge and heat diffusivities in momentum dissipating holography
}

\author{
Andrea Amoretti, ${ }^{a, b, e}$ Alessandro Braggio, ${ }^{b, c}$ Nicodemo Magnoli ${ }^{a, b}$ and Daniele Musso ${ }^{d}$ \\ ${ }^{a}$ Dipartimento di Fisica, Università di Genova, \\ via Dodecaneso 33, I-16146, Genova, Italy \\ ${ }^{b}$ INFN - Sezione di Genova, \\ via Dodecaneso 33, I-16146, Genova, Italy \\ ${ }^{c}$ CNR-SPIN, \\ Via Dodecaneso 33, 16146, Genova, Italy \\ ${ }^{d}$ Abdus Salam International Centre for Theoretical Physics (ICTP), \\ Strada Costiera 11, I-34151 Trieste, Italy \\ ${ }^{e}$ Lorentz Institute for Theoretical Physics, \\ Niels Bohrweg 2, Leiden NL-2333 CA, The Netherlands \\ E-mail: andrea.amoretti@ge.infn.it, alessandro.braggio@spin.cnr.it, \\ nicodemo.magnoli@ge.infn.it, dmusso@ictp.it
}

ABSTRACT: Inspired by a recently conjectured universal bound for thermo-electric diffusion constants in quantum critical, strongly coupled systems and relying on holographic analytical computations, we investigate the possibility of formulating Planckian bounds in different holographic models featuring momentum dissipation. For a certain family of solutions to a simple massive gravity dilaton model at zero charge density we find linear in temperature resistivity and entropy density alongside a constant electric susceptibility. In addition we explicitly find that the sum of the thermo-electric diffusion constants is bounded.

KEYwords: AdS-CFT Correspondence, Holography and condensed matter physics (AdS/CMT)

ARXIV EPRINT: 1411.6631 


\section{Contents}

1 Introduction 1

2 The simplest holographic model: massive gravity 3

3 Diffusion constants $\quad 5$

4 Adding the dilaton $\quad 7$

5 Analysis at criticality $\quad 8$

$\begin{array}{llr}6 & \text { Discussion } & 11\end{array}$

\section{Introduction}

The description of universal behaviors of strongly correlated systems despite the differences in their microscopic structure is challenging and of high physical relevance. One of the best known examples is the linear-in-temperature resistivity regime shared by many apparently very different materials like cuprates, heavy fermions, pnictides, ruthenates and fullurenes [1]. In recent times, a great effort has been put into characterizing the universal behavior of these materials by determining bounds on appropriate physical quantities. The reason why this approach is promising is twofold: first, a bound can be formulated independently of the microscopic detail of the system and can therefore aspire to be universal; secondly, in an incoherent quantum critical regime, the system equilibrates on a time scale independent of any microscopic energy scale and just determined by the temperature. Therefore, as a consequence of the insensitivity to energy scales other than the temperature, incoherent strongly correlated systems may tend to saturate general temperaturedependent bounds.

Strongly correlated models amenable to quantitative theoretical control are extremely rare. In this respect, the gauge/gravity duality is very useful since it provides a dual description for some strongly interacting quantum field theories where equilibrium and transport properties can be computed. Actually, the celebrated proposal of a universal bound for momentum diffusion in strongly coupled systems was advanced relying on holography. This bound was originally conjectured in [2] in terms of the ratio of the shear viscosity to the entropy density, namely $\eta / s \geq \hbar /\left(4 \pi k_{B}\right)$. The approximate saturation of the viscosity bound has been subsequently observed in the quark gluon plasma created at the heavy ion colliders, as well as in the unitary fermionic cold atom gas [3]. Moreover, recent experimental ARPES measurements on an optimally doped cuprate show that, also in this case, the ratio $\eta / s$ due to the intrinsic electronic contribution is close to saturate the afore mentioned bound [4]. This experimental evidence strongly suggests that the transport properties of 
the strange metals due to the strongly correlated electron behavior could be described in the holographic framework.

The physical origin of the $\eta / s$ bound can be explained referring to the concept of "Planckian" dissipation $[5,6]$ : the fact that, in the finite temperature quantum critical state, physical quantities relax extremely rapidly and the equilibration rate is substantially determined by the uncertainty principle, namely $\tau_{\text {eq }} \sim \hbar /\left(k_{B} T\right)$. Recent experimental observations showing that a wide range of strange metals in the linear-in-temperature resistivity regime present an equilibration rate of this kind [1] strongly supports the idea that a unified framework might capture the properties of these strongly interacting materials.

Relying on a wider applicability of similar arguments, it is tempting to find whether other physical observables in strange metals and, more generally, in strongly correlated materials have to saturate a bound due to Planckian dissipation. However, the identification of the correct kinematic framework where one can formulate universal bounds is, in general, not easy. The reason being that in metallic materials the mechanisms for momentum relaxation, such as the lattice or scattering from disorder, which are necessary to make the conductivities finite, are extrinsic to the electron dynamics. Hence it is difficult to formulate intrinsic and universal bounds.

A recent proposal advanced in [7] suggested that, in analogy to the bound on $\eta / s$, the quantities that could be universally bounded in strange metals are the thermo-electric diffusion constants $D_{+}$and $D_{-}$. More specifically in [7] it was argued that strongly correlated metals in the incoherent regime (where momentum is quickly degraded) have charge and heat diffusion constants that eventually saturate the bound

$$
D_{ \pm} \geq C \frac{\hbar \bar{v}^{2}}{k_{B} T}
$$

where $C$ is an undetermined constant and $\bar{v}$ is a characteristic velocity of the critical and relativistically invariant system. ${ }^{1}$ This velocity represents an extra scale of the relativistic low-energy effective description which may be naturally related to a UV cut-off (as described for instance in [8]). It is relevant to note that, in the holographic context, an extra scale of the effective description was for instance used in [9] in order to set two different temperature scalings for the resistivity and the Hall angle.

The diffusion constants $D_{+}$and $D_{-}$are related to the transport coefficients via the Einstein relations, namely:

$$
\begin{aligned}
D_{+} D_{-} & =\frac{\sigma}{\chi} \frac{\kappa}{c_{\rho}} \\
D_{+}+D_{-} & =\frac{\sigma}{\chi}+\frac{\kappa}{c_{\rho}}+\frac{T(\zeta \sigma-\chi s)^{2}}{c_{\rho} \chi^{2} \sigma},
\end{aligned}
$$

where $\sigma, s$ and $\kappa$ are respectively the electric, the thermo-electric and the thermal conductivities (see [7] for a derivation); $c_{\rho}$ is the specific heat at fixed charge density $\rho, \zeta$ is the thermo-electric susceptibility and $\chi$ is the electric susceptibility. The bounds (1.1)

\footnotetext{
${ }^{1}$ We avoid referring to $\bar{v}$ as a Fermi velocity strictly speaking because the latter does not admit in general a sharp definition in strongly correlated systems.
} 
can therefore be translated in terms of the thermo-electric transport coefficients of the model. ${ }^{2}$ In particular, relying on qualitative arguments, ${ }^{3}$ in [7] it was suggested that incoherent metals approximatively saturating the bounds (1.1) have a linear-in-temperature resistivity controlled precisely by the equilibration time-scale $\tau_{\text {eq }} \sim \hbar /\left(k_{B} T\right)$.

Soon after the analysis of [7], the author of [8], relying on hydrodynamical arguments, suggested that a bound on the sum of the thermoelectric diffusion constants is more natural than bounding the same diffusivities individually, namely

$$
D_{+}+D_{-} \geq C^{\prime} \frac{\hbar \bar{v}^{2}}{k_{B} T}
$$

still [8] (to which we refer for details) observes that the sum $D_{+}+D_{-}$is always a real quantity and argues on the basis of a perturbative approach.

In this paper we first study the charge and heat diffusion in the simplest holographic model featuring spatial diffeomorphism breaking by means of a mass term for the graviton and argue that its dynamics is not suitable to feature linear in $T$ resistivity and diffusion bounds. For this reason we consider a generalized model comprehending also a dilaton field. We first show that a particular family of solutions to a massive gravity dilaton model has interesting features (among which, notably, a linear in $T$ resistivity) and remarkably we find that the sum of the diffusion constants is bounded in line with [8].

The key point and novelty of the present analysis consists in considering the massive gravity dilaton model at vanishing chemical potential but with a non-trivial dilaton profile. This is essential to lead to linear in $T$ resistivity in the whole temperature range. Such linear in $T$ behavior of the resistivity is robust also with respect to the momentum dissipation physics meaning that its linearity in $T$ proves insensitive to the specific strong or weak momentum dissipation regime.

\section{The simplest holographic model: massive gravity}

Our computations rely on the phenomenologically motivated holographic model [11] which features massive gravitons in the dual gravitational $3+1$ dimensional bulk. The UV derivation and ultimate consistency of massive gravity are still open questions which, to the present purposes, can be set aside as long as the phenomenology of the $2+1$ dimensional boundary theory is itself consistent. This was proven to be the case both in relation to the thermodynamics [14] and on the level of the thermo-electric transport properties [16].

The gravitational action is

$$
S=\int d^{4} x \sqrt{-g}\left[R+\frac{6}{L^{2}}+\mathcal{M}(g)-\frac{1}{4} F_{\mu \nu} F^{\mu \nu}\right]+S_{\text {c.t. }},
$$

\footnotetext{
${ }^{2}$ As observed in [28], we underline the fact that the definitions of individual quantities like $\sigma$ or $\eta$ are sensitive to the normalization of the associated current. For the purpose of formulating universal statements is then crucial to consider quantities which are independent of normalization choices as the ratios giving rise to the diffusivities in (1.2) and (1.3). Note that, in line with Onsager's symmetry argument about the thermo-electric conductivity matrix (namely $s$ represents both the thermo-electric and the electro-thermal entries), the normalization of the heat flow is not independent of that of the electric current.

${ }^{3}$ Specifically on the assumption that susceptibilities are essentially temperature independent in the relevant regime.
} 
where $L$ is the asymptotic $A d S_{4}$ radius and

$$
\mathcal{M}(g)=\alpha \operatorname{tr}(\mathcal{K})+\beta\left[\operatorname{tr}(\mathcal{K})^{2}-\operatorname{tr}\left(\mathcal{K}^{2}\right)\right],
$$

represents the graviton mass term expressed in terms of the matrix $\mathcal{K}$ whose definition is $\mathcal{K}^{\mu}{ }_{\nu} \equiv\left(\sqrt{\mathcal{K}^{2}}\right)^{\mu}{ }_{\nu}$ with $\left(\mathcal{K}^{2}\right)^{\mu}{ }_{\nu} \equiv g^{\mu \rho} f_{\rho \nu}$ and the two parameters $\alpha$ and $\beta$ having both dimension of mass ${ }^{2}$. The matrix $f$ is a non-dynamical auxiliary metric whose explicit form is $f_{\mu \nu}=\operatorname{diag}(0,0,1,1)$. The action (2.1) contains also the Maxwell-Einstein term (with $F=d A$ ) and a boundary term $S_{\text {c.t. }}$ (see [16] for the proper definition of the latter) necessary to have a well-defined variational problem and a finite on-shell action. Henceforth we consider $L=1$ since this is just a choice of unit of measure .

Following the standard holographic dictionary, it is known that (2.1) is the gravitational dual of a strongly correlated system with charged degrees of freedom exhibiting an extrinsic elastic mechanism of momentum dissipation such as that generated by the presence of quenched disorder $[11,14-16]$. The model admits planar black brane solutions of this kind

$$
d s^{2}=\frac{1}{z^{2}}\left[-f(z) d t^{2}+d x^{2}+d y^{2}+\frac{1}{f(z)} d z^{2}\right], \quad A=a(z) d t
$$

where the explicit functions appearing in the ansatz are

$$
a(z)=\mu\left(1-\frac{z}{z_{h}}\right), \quad f(z)=1+z^{2} \beta-\frac{z^{3} \beta}{z_{h}}-\frac{z^{3}}{z_{h}^{3}}+\frac{z \alpha}{2}-\frac{z^{3} \alpha}{2 z_{h}^{2}}-\frac{z^{3} \mu^{2}}{4 z_{h}}+\frac{z^{4} \mu^{2}}{4 z_{h}^{2}},
$$

where $z_{h}$ is the horizon radius defined by $f\left(z_{h}\right)=0$ and $\mu$ is the chemical potential of the dual quantum field theory. According to the holographic dictionary, this black brane solution is dual to finite temperature equilibrium states of the boundary theory whose thermodynamic variables are encoded in the parameters and quantitative details of the bulk background, namely

$$
T=\frac{1}{4 \pi z_{h}}\left(3+z_{h} \alpha+z_{h}^{2} \beta-\frac{z_{h}^{2} \mu^{2}}{4}\right), \quad \mathcal{S}=\frac{4 \pi}{z_{h}^{2}}, \quad \rho=\frac{\mu}{z_{h}},
$$

where $T$ is the temperature, $\mathcal{S}$ is the entropy density and $\rho$ is the charge density.

The parameter $\alpha$ and $\beta$ control the strength of the momentum dissipation mechanism, namely the momentum is dissipated faster as $|\alpha|$ and $|\beta|$ increase. In particular, in the coherent regime, where momentum is slowly dissipated, an extrinsic dissipation rate $\tau_{\text {ext }}^{-1}$ can be defined [15]:

$$
\tau_{\mathrm{ext}}^{-1}=-\frac{4\left(\alpha+2 \beta z_{h}\right)}{12+3 z_{h}^{2} \mu^{2}}
$$

In order to obtain the diffusion constants from the system of equations (1.2) and (1.3), we need at first the explicit expressions of the DC transport coefficients. An analytical computation (see [17] for the case $\alpha=0$ ) leads to

$$
\sigma=1-\frac{z_{h} \mu^{2}}{\alpha+2 z_{h} \beta}, \quad s=\frac{4 \pi \mu}{\alpha+2 z_{h} \beta}, \quad \kappa=-\frac{\pi\left[z_{h}^{2}\left(4 \beta-\mu^{2}\right)+4 z_{h} \alpha+12\right]}{z_{h}^{2}\left(\alpha+2 z_{h} \beta-z_{h} \mu^{2}\right)} ;
$$


secondly, we have to compute $c_{\rho}, \chi$ and $\zeta$ for the model at hand. This can be easily achieved using their thermodynamic definitions, namely

$$
\begin{gathered}
\chi=\left(\frac{\partial \rho}{\partial \mu}\right)_{T}=\frac{z_{h}^{2}\left(3 \mu^{2}-4 \beta\right)+12}{z_{h}^{3}\left(\mu^{2}-4 \beta\right)+12 z_{h}}, \\
\zeta=\left(\frac{\partial \rho}{\partial T}\right)_{\mu}=\frac{16 \pi \mu}{z_{h}^{2}\left(\mu^{2}-4 \beta\right)+12}, \\
c_{\rho}=T\left(\frac{\partial \mathcal{S}}{\partial T}\right)_{\mu}-\frac{\zeta^{2} T}{\chi}=-\frac{8 \pi\left[z_{h}^{2}\left(4 \beta-\mu^{2}\right)+4 z_{h} \alpha+12\right]}{z_{h}^{4}\left(4 \beta-3 \mu^{2}\right)-12 z_{h}^{2}},
\end{gathered}
$$

where, in order to perform the thermodynamic derivatives with respect to $T$ and $\mu$ one has to keep into account that $z_{h}$ is a function of both the temperature and the chemical potential due to the first of the relations (2.5). It is interesting to note that (2.8) and (2.9) do not explicitly depend on $\alpha$ (they do so only through $z_{h}$ ); formally they have the same expression as in the $\alpha=0$ case [17].

\section{Diffusion constants}

Once both the thermodynamics and the linear response of the system are under control, it is possible to study the diffusion constants through the Einstein relations (1.2) and (1.3). At first we will consider the simplest case in which the parameter $\alpha$ is set to zero, and we will comment on the general case at the end of the section. The diffusion constants for $\alpha=0$ take the following form

$$
D_{ \pm}=\frac{-12+z_{h}^{2}\left(20 \beta-19 \mu^{2}\right) \pm \sqrt{\Delta}}{32 \beta z_{h}}
$$

where

$$
\Delta=z_{h}^{4}\left(144 \beta^{2}-696 \beta \mu^{2}+361 \mu^{4}\right)+z_{h}^{2}\left(288 \beta+456 \mu^{2}\right)+144 .
$$

We refer to figure 1 to have a qualitative idea of the generic behaviour of the diffusion constants with respect to the temperature. In the simple case of zero chemical potential $\mu=0$ (see right panel of 1 ) the electric and thermal sector decouple and the two diffusivities take the following form

$$
\begin{aligned}
& D_{c}=\frac{\sigma}{\chi}=-\frac{\sqrt{4 \pi^{2} T^{2}-3 \beta}-2 \pi T}{\beta}, \\
& D_{h}=\frac{\kappa}{c_{\rho}}=-\frac{\sqrt{4 \pi^{2} T^{2}-3 \beta}}{\beta} .
\end{aligned}
$$

We observe that in the high temperature regime $D_{c}$ has an $1 / T$ behavior; this holds true also when $\alpha \neq 0$. As we will comment later, $\beta$ is negative. Even though also for $\mu \neq 0$ the diffusivities are bounded from below by an $1 / T$ power law in the high temperature region, the coefficient of such bounding behavior can be apparently lowered at will acting on the mass parameter $\beta$. Nevertheless, to be precise on this point, one needs to consider whether 

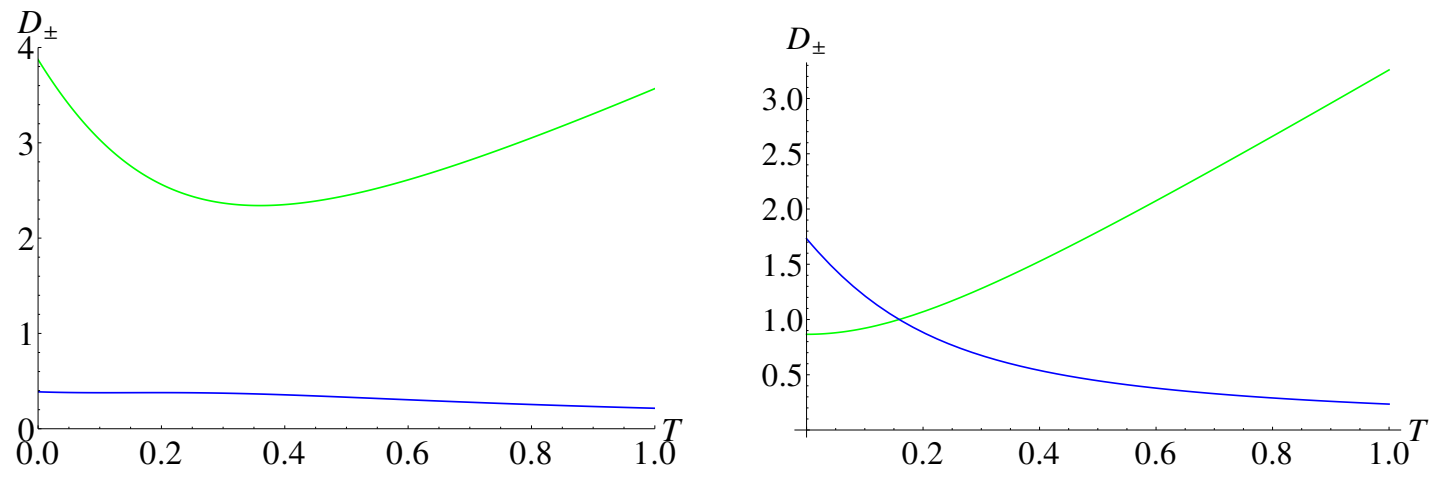

Figure 1. Sample diagrams to illustrate the behavior of thermo-electric diffusion constants at (left) finite chemical potential, namely $\mu=1$ (blue line for $D_{-}$and green line for $D_{+}$), and (right) $\mu=0$ (blue line for $D_{-}=D_{c}$ and green line for $D_{+}=D_{h}$ ). The graviton mass parameter has been chosen to be $\beta=-1$.

the range of $\beta$ is possibly limited by consistency requirements of the model in such a way to produce a lower bound for the conductivities.

By means of simple manipulations, based on the expression for $T$ in (2.5), it is possible to show that demanding a positive momentum dissipation rate $\tau_{\text {ext }}^{-1}(2.6)$ (with $\alpha=0$ ) implies

$$
\beta<0 \text {. }
$$

A further consistency requirement is provided by asking the positivity of the energy density. More specifically, we consider the holographic renormalization of the model (2.1) on the black brane solutions (2.4) and the assumption that finite counter-terms do not affect the thermodynamics (namely that they do not depend on the thermodynamic variables) as discussed in [14]. It is then possible to refer the renormalized energy density to its value at zero $T$ (keeping fixed all the other quantities) and ask that its value at finite $T$ be never lower than that at $T=0$, namely

$$
\mathcal{E}(T, \mu ; \beta)-\mathcal{E}(0, \mu ; \beta)>0 .
$$

In this $\alpha=0$ case, this energy requirement does not furnish any additional constraint on the parameter $\beta$, and the renormalized energy density appearing in (3.6) remains positive for every $\beta<0$.

All in all, the consistency requirements, although restricting the possible choices of mass parameters, do not lead either to a diffusion bound nor to a minimal value for the diffusion constants below which the holographic model is not trustworthy. Indeed it is always possible to achieve a strongly incoherent regime (where momentum is quickly dissipated) by sending $|\beta|$ to infinity without any apparent consistency problem.

In line with these observations, one has that the infinite $|\beta|$ limit leads to vanishing diffusion constants, namely

$$
\lim _{\beta \rightarrow-\infty} D_{ \pm}(T, \beta)=0
$$

Despite this limit corresponds to a bulk model dominated just by the mass term of the graviton where the dynamics of the metric vector fluctuation of interest trivializes, such 
argument does not yield a quantitative ground to formulate a bound. Even observing that an infinite $|\beta|$ limit leads necessary to a regime where the graviton mass exceeds the Planck mass one could limit the value of the gravity model as an effective field theory rather than supporting the existence of a bound on diffusivity.

Extending to the case where $\alpha \neq 0$, the mass of the graviton is given by

$$
m^{2}(z)=-2 \beta+\frac{\alpha}{z}
$$

which diverges in the UV region. We have performed the same analysis illustrated for $\alpha=0$ also for the $\alpha \neq 0$ case. Even though the analysis is technically more involved, there are no qualitative differences and, for every fixed values of $\alpha$, the incoherent regime can be obtained by performing the limit $|\beta| \rightarrow \infty$ leading to unbounded diffusion constants as in (3.7).

The outcome of the previous analyses is that the simple massive gravity model (2.1) does not allow to formulate consistent bounds on the thermo-electric diffusion constants even in the case where the mass of the graviton has non-trivial RG (i.e. radial) behavior. ${ }^{4}$ In addition it is important to observe that the electric conductivity (2.7) is, in a regime of large $|\beta|$, dominated by the constant term, namely the charge conjugation symmetric piece. This highlights a limitation in reproducing a linear in $T$ resistivity in massive gravity in a regime where momentum dissipation exceeds the other scales of the system. In other words, the incoherent regime considered to formulate the conjecture on diffusion bounds (phenomenologically motivated by the consequent linear in $T$ resistivity $^{5}$ ) does not lead to the desired phenomenology in the simple massive gravity model (2.1) and, as we will see in the next sections, an analysis of the diffusion constants in a model where these requirements are fulfilled is mandatory.

\section{Adding the dilaton}

We consider a slight generalization of the massive gravity model (2.1). We introduce a dilaton and consider the following bulk action

$$
S_{d}=\int d^{4} x \sqrt{-g}\left[R+6 \cosh \phi-\frac{e^{\phi}}{4} F_{\mu \nu} F^{\mu \nu}-\frac{3}{2} \partial_{\mu} \phi \partial^{\mu} \phi+\mathcal{M}_{\beta}(g)\right]+S_{\text {c.t. }},
$$

where the mass term we consider is given $b^{6}$

$$
\mathcal{M}_{\beta}(g)=\beta\left[\operatorname{tr}(\mathcal{K})^{2}-\operatorname{tr}\left(\mathcal{K}^{2}\right)\right] .
$$

This model is inspired by [21] which was considered in $3+1$ dimensions with a mass term for the graviton in [22]. This dilaton theory is particularly appealing as it features linear

\footnotetext{
${ }^{4}$ Recently a more general mass potential for the graviton has been taken into account [30]; even though it seems that no qualitative differences occur in this case, it would be interesting to perform here a similar analysis of the diffusion constants.

${ }^{5}$ See [7] for further details and assumptions, such as the insensitivity of the electric susceptibility $\chi$ to temperature.

${ }^{6}$ Namely the same as considered previously in (2.2) fixing $\alpha=0$.
} 
in $\mathrm{T}$ entropy at low temperature, characteristics which was at the core of the argument described in [22] to have linear in $T$ resistivity at low temperature $(T / \mu)$. In addition, as opposed to (2.1), we have here no residual entropy at zero temperature.

The bulk solutions of (4.1) are

$$
\begin{aligned}
d s^{2} & =\frac{g(z)}{z^{2}}\left(-h(z) d t^{2}+\frac{d z^{2}}{g(z)^{2} h(z)}+d x^{2}+d y^{2}\right), \\
A_{t} & =\sqrt{\frac{3 Q\left(Q z_{h}+1\right)}{z_{h}}\left(1+\frac{\beta z_{h}^{2}}{\left(Q z_{h}+1\right)^{2}}\right)} \frac{z_{h}-z}{z_{h}(Q z+1)}, \\
\phi(z) & =\frac{1}{3} \log g(z), \quad g(z)=(1+Q z)^{\frac{3}{2}}, \\
h(z) & =1+\frac{\beta z^{2}}{(Q z+1)^{2}}-\frac{z^{3}\left(Q z_{h}+1\right)^{3}}{z_{h}^{3}(Q z+1)^{3}}\left(1+\frac{\beta z_{h}^{2}}{\left(Q z_{h}+1\right)^{2}}\right) .
\end{aligned}
$$

Where the parameter $Q$ controls the dilaton profile and is related to the chemical potential.

The thermodynamics of the model is holographically related to the bulk solution in the following way

$$
\begin{aligned}
T & =\frac{3\left(1+Q z_{h}\right)^{2}+\beta z_{h}^{2}}{4 \pi\left(1+Q z_{h}\right)^{\frac{3}{2}} z_{h}}, \quad \mathcal{S}=\frac{4 \pi}{z_{h}^{2}}\left(Q z_{h}+1\right)^{\frac{3}{2}}, \\
\mu & =\sqrt{\frac{3 Q\left(Q z_{h}+1\right)}{z_{h}}\left[1+\frac{\beta z_{h}^{2}}{\left(Q z_{h}+1\right)^{2}}\right]} \\
\rho & =\frac{\mu}{z_{h}}\left(Q z_{h}+1\right),
\end{aligned}
$$

Relying again on analytical computation (still along the lines of a generalization of the membrane paradigm described in [18]) we have analytical control upon the entire set of thermo-electric transport coefficients and obtain the following explicit expressions

$$
\begin{aligned}
\sigma & =\frac{2 \beta\left(Q z_{h}+1\right)-3 Q z_{h}\left[\beta+\left(\frac{1}{z_{h}}+Q\right)^{2}\right]}{2 \beta \sqrt{Q z_{h}+1}}, \\
s & =-\frac{2 \sqrt{3} \pi}{\beta z_{h}} \sqrt{Q\left(Q z_{h}+1\right)\left[Q\left(Q z_{h}+2\right)+\beta z_{h}+\frac{1}{z_{h}}\right]}, \\
\kappa & =\frac{4 \pi\left(Q z_{h}+1\right)\left[3\left(Q z_{h}+1\right)^{2}+\beta z_{h}^{2}\right]}{z_{h}^{2}\left[\beta z_{h}\left(Q z_{h}-2\right)+3 Q\left(Q z_{h}+1\right)^{2}\right]} .
\end{aligned}
$$

We remind the reader that, also in the present dilaton model, the positivity of the momentum dissipation rate requires $\beta<0$ so in the preceding formulæ $\beta$ is always negative.

\section{Analysis at criticality}

The common structure of the cuprate phase diagram (see for instance [32]) shows that the strange metal phase is reached, in general, at high $T$. Given the scaling properties of the 
holographic model with respect to a rescaling of the boundary space-time, ${ }^{7}$ it is natural to compare the temperature to the chemical potential and consider the scaling invariant ratio $T / \mu$. Hence we mean high temperatures in the sense $T / \mu \gg 1$ and, inverting the qualitative argument just given, it is natural to study the $\mu=0$ case to describe the "criticality" condition which gives rise to the strange metal. On top of this, when the chemical potential is vanishing, the charge and heat transport of the model decouple.

Asking for $\mu=0$ in (4.4) we face two possibilities. We can set $Q=0$ and consequently trivialize the dilaton profile falling back to the solution (2.4) already found in simple massive gravity. Alternatively we can set

$$
Q z_{h}+1=|\beta|^{1 / 2} z_{h}
$$

we focus on this second case where the dilaton has non-trivial radial profile. For completeness, let us present explicitly the background solutions in this case,

$$
\begin{array}{rlrl}
d s^{2} & =\frac{g(z)}{z^{2}}\left(-h(z) d t^{2}+\frac{d z^{2}}{g(z)^{2} h(z)}+d x^{2}+d y^{2}\right), \\
A_{t} & =0, \quad \phi(z)=\frac{1}{3} \log g(z), \\
g(z) & =\left(1+|\beta|^{1 / 2} z-\frac{z}{z_{h}}\right)^{\frac{3}{2}}, & h(z)=1+\frac{\beta z^{2} z_{h}^{2}}{\left(z_{h}+|\beta|^{1 / 2} z z_{h}-z\right)^{2}} .
\end{array}
$$

As we will see shortly, enforcing relation (5.1) is far more than a technicality; indeed it corresponds to considering non-trivial critical conditions which relate the parameter $Q$ to the other scales of the model (the temperature, through $z_{h}$, and the graviton mass $\beta$ ) in a particular manner. Fixing $\mu=0$ by imposing relation (5.1) defines a family of backgrounds whose phenomenology differs from that of simple massive gravity. As we are going to describe precisely, the new features of such a family present a linear in $T$ resistivity and an overall physical behavior in line with that indicated in [7] as the basis for discussing diffusion bounds. ${ }^{8}$ Let us further underline that (5.1) and $Q=0$ define two different branches of possible backgrounds that have different physical properties.

Studying the thermodynamics of the critical conditions associated to (5.1) leads to

$$
T=\frac{|\beta|^{1 / 4}}{2 \pi z_{h}^{1 / 2}}, \quad \rho=0, \quad \mathcal{S}=8 \pi^{2}|\beta|^{1 / 2} T,
$$

which feature linear in $T$ entropy at all temperature. Moreover, regarding the susceptibilities, we obtain

$$
\begin{aligned}
\zeta & =0, \\
\chi & =|\beta|^{1 / 2}, \\
c_{\rho} & =8 \pi^{2}|\beta|^{1 / 2} T .
\end{aligned}
$$

\footnotetext{
${ }^{7}$ See for instance [16] for the analysis of the scaling properties of the pure massive gravity model.

${ }^{8}$ We postpone the investigation of the a priori reasons of such an interesting physical behavior and a possible detailed interpretation of the boundary theory dual to the dilaton model to future work. A relevant study in this sense (although performed in massless gravity) is described in [21].
} 
Particularly interesting to us is the electric susceptibility $\chi$ independent from the temperature. Continuing the analysis of the critical model resulting from the condition (5.1), we find the transport coefficients to be

$$
\begin{aligned}
\sigma & =|\beta|^{1 / 4} z_{h}^{1 / 2} \Longrightarrow \sigma^{-1}=2 \pi|\beta|^{-1 / 2} T, \\
s & =0 \\
\kappa & =16 \pi^{3}|\beta|^{-1 / 2} T^{2} .
\end{aligned}
$$

We underline the fact that the resistivity is linear in $T$ for the entire range of temperature.

The set of equilibrium and transport quantities just found fulfills precisely the phenomenological framework that was considered in formulating diffusion bounds in [7]. More specifically, a constant electric susceptibility was assumed as a hypothesis and a linear in $T$ resistivity as the consequence of an electrical diffusivity saturating the bound. Moreover, in [7] it was conjectured that the charge diffusion constant $D_{c}$ obeys a bound which depends only on temperature; the arguments relying on the hypothesis that the momentum dissipation rate is fast with respect to the scale of $T$, a regime referred to as incoherent regime.

Analyzing the dilaton model we find that, in the critical condition associated to (5.1), the charge diffusion constant takes a very simple form independent on the parameter $\beta$, namely

$$
D_{c}^{\text {(crit) }}=\frac{\sigma}{\chi}=\frac{1}{2 \pi T} .
$$

It is very important to note that (5.10) (emerging from (5.3)-(5.6) and (5.7)) does not refer to any specific regime for the momentum dissipation rate controlled by $\beta$; in this sense, it is not necessarily related to either an incoherent or a coherent regime. Such feature is very appealing because allows us to be "agnostic" about the hierarchy of $T$ and the momentum dissipating scale $|\beta|$; the phenomenology of the critical model at hand is therefore robust also in this sense.

Let us now focus on the heat diffusion constant $D_{h}$. The assumptions of constant susceptibility $\chi$ and linear in $T$ resistivity do not suggest a bound for $D_{h}$ which, still following [7], can nevertheless be conjectured by analogy with $D_{c}$. For the critical dilaton model $D_{h}$ takes the following explicit form

$$
D_{h}^{(\mathrm{crit})}=\frac{\kappa}{c_{\rho}}=\frac{2 \pi T}{|\beta|} .
$$

In order to discuss the possibility of formulating a bound, let us consider the incoherent regime where momentum is dissipated quickly. As for the simple massive gravity model considered in section 3 , the incoherent regime is achieved in the limit $T /|\beta| \rightarrow 0$. The heat diffusion constant (5.11) depends explicitly on $\beta$ while instead the charge diffusion constant (5.10) does not. This crucial difference leads to the impossibility to rely on incoherence and formulate a lower bound which depends only on temperature for $D_{h}^{(\text {crit })}$.

Although one cannot formulate a bound for both the heat and charge diffusion constants separately, as a direct consequence of the bound on $D_{c}^{\text {(crit) }}$, the sum of the two is naturally bounded once incoherence is considered, namely

$$
D_{h}^{\text {(crit) }}+D_{c}^{\text {(crit) }} \geq \frac{1}{2 \pi T} .
$$


In [8] different arguments were proposed to motivate a bound on the sum of diffusion constants rather than on them individually relying on a hydrodynamical analysis. We refer to [8] for a detailed discussion on the topic but intuitively we note that the quantity $D_{h}+D_{c}$ is more natural to formulate a bound since in general the two diffusion constants separately could be a complex quantities while their sum is always real.

The present analysis of the holographic dilaton model moves somehow oppositely with respect to [8]: we were able to compute the two diffusion constants separately and we have found that a bound can be formulated only on the sum of the two once incoherence is considered.

The set of results obtained through the study of the critical massive gravity dilaton model, and specifically (5.12), appear to be intimately related to the detail of the model. Actually, as we have seen explicitly, simple massive gravity led to different results. At the best of our knowledge, the dilaton model under consideration studied at $\mu=0$ according to (5.1) is the only holographic model were all the assumptions made in [7] are fulfilled. It is therefore particularly relevant that in such circumstances a bound on the sum of the diffusion constants can be naturally formulated. In other words it is possible to consider the present analysis as a support (at least in a specific and well defined case) of the physics conjectured in [7].

\section{Discussion}

In this paper we have analyzed heat and thermal diffusion constants in two different models exhibiting momentum dissipation realized by means of a bulk massive graviton. In the simplest holographic massive gravity theory [11] we find that diffusion constants are not bounded from below and, more precisely, vanish in the incoherent limit where momentum is dissipated quickly. We also show that a careful analysis of the graviton mass parameter space constrained by physical consistency requirements (such as a positive momentum dissipation rate and a positive energy density) does not allow to bound the diffusivities and neither to give a lower diffusion value beyond which the model becomes unreliable. These results are not surprising when confronted with the framework adopted by [7] to propose a conjecture on diffusion bounds. Indeed, the conjecture itself is formulated in relation to phenomenological properties like a linear in $T$ resistivity and a constant electric susceptibility which are not realized by the simplest massive gravity model.

To pursue the study on diffusion appears to be necessary to enrich the holographic model. Hence we consider the addition of a dilaton field as in [22]. We showed that a subfamily of solutions to this dilaton theory corresponding to specific critical conditions reproduces the desired phenomenological behavior, $\sigma^{-1} \sim T$ and constant $\chi$, at all values of the temperature. This constitutes a relevant result by itself.

We pursue the analysis of diffusion for the dilaton model to check the connection between transport features and diffusion bounds suggested in [7]. The critical dilaton model leads to a diffusion bound (of the conjectured $1 / T$ form) in the charge sector while featuring an unbounded heat diffusion constant. These picture can be connected to the study performed in [8] where, on general grounds, it is argued in favor on a diffusion bound 
on the sum of the thermo-electric diffusion constants rather than on them individually. In conclusion, the present analysis of the critical massive gravity dilaton model offers support to both the proposal of diffusion bounds advanced in [7] and [8].

\section{Acknowledgments}

A particular thank goes to R. Davison for crucial discussions on the first version of the paper. We would also like to thank R. Argurio, M. Bertolini, M. Carrega, A. Dabholkar, N. Maggiore, A. Mezzalira, N. Pinamonti and G. Villadoro. D.M. would also like to thank F. Bigazzi, P. Creminelli, N. Iqbal, D. Forcella, L. Pando Zayas, N. Pinzani-Fokeeva, G. Policastro, F. Porri, D. Redigolo and D. T. Son for very nice and insightful discussions. A.A. acknowledges support of a grant from the John Templeton foundation. The opinions expressed in this publication are those of the authors and do not necessarily reflect the views of the John Templeton foundation. A. B. thanks the MIUR-FIRB2012 — Project HybridNanoDev (Grant No. RBFR1236VV) and European Union FP7/2007-2013 under REA grant agreement no 630925 - COHEAT.

Open Access. This article is distributed under the terms of the Creative Commons Attribution License (CC-BY 4.0), which permits any use, distribution and reproduction in any medium, provided the original author(s) and source are credited.

\section{References}

[1] J.A.N. Bruin, H. Sakai, R.S. Perry and A.P. Mackenzie, Similarity of scattering rates in metals showing T-linear resistivity, Science 339 (2013) 804.

[2] P. Kovtun, D.T. Son and A.O. Starinets, Viscosity in strongly interacting quantum field theories from black hole physics, Phys. Rev. Lett. 94 (2005) 111601 [hep-th/0405231] [INSPIRE].

[3] A. Adams et al., Strongly correlated quantum fluids: ultracold quantum gases, quantum chromodynamic plasmas and holographic duality, New J. Phys. 14 (2012) 115009 [arXiv:1205.5180] [INSPIRE].

[4] J.D. Rameau et al., Nearly perfect fluidity in a high temperature superconductor, Phys. Rev. B 90 (2014) 134509 [arXiv: 1409.5820] [InSPIRE].

[5] S. Sachdev, Quantum phase transitions, Cambridge University Press, Cambridge U.K. (1999).

[6] J. Zaanen, Superconductivity: why the temperature is high, Nature 430 (2004) 512.

[7] S.A. Hartnoll, Theory of universal incoherent metallic transport, Nature Phys. 11 (2015) 54 [arXiv: 1405.3651] [INSPIRE].

[8] P. Kovtun, Fluctuation bounds on charge and heat diffusion, J. Phys. A 48 (2015) 265002 [arXiv: 1407.0690] [INSPIRE].

[9] M. Blake and A. Donos, Quantum critical transport and the Hall angle, Phys. Rev. Lett. 114 (2015) 021601 [arXiv: 1406.1659] [INSPIRE].

[10] S. Cremonini, U. Gürsoy and P. Szepietowski, On the temperature dependence of the shear viscosity and holography, JHEP 08 (2012) 167 [arXiv:1206.3581] [INSPIRE]. 
[11] D. Vegh, Holography without translational symmetry, arXiv:1301.0537 [INSPIRE].

[12] L. Alberte and A. Khmelnitsky, Stability of massive gravity solutions for holographic conductivity, Phys. Rev. D 91 (2015) 046006 [arXiv:1411.3027] [InSPIRE].

[13] T. Andrade and B. Withers, A simple holographic model of momentum relaxation, JHEP 05 (2014) 101 [arXiv:1311.5157] [INSPIRE].

[14] M. Blake and D. Tong, Universal resistivity from holographic massive gravity, Phys. Rev. D 88 (2013) 106004 [arXiv:1308.4970] [INSPIRE].

[15] R.A. Davison, Momentum relaxation in holographic massive gravity, Phys. Rev. D 88 (2013) 086003 [arXiv: 1306.5792] [INSPIRE].

[16] A. Amoretti, A. Braggio, N. Maggiore, N. Magnoli and D. Musso, Thermo-electric transport in gauge/gravity models with momentum dissipation, JHEP 09 (2014) 160 [arXiv: 1406.4134] [INSPIRE].

[17] A. Amoretti, A. Braggio, N. Maggiore, N. Magnoli and D. Musso, Analytic dc thermoelectric conductivities in holography with massive gravitons, Phys. Rev. D 91 (2015) 025002 [arXiv: 1407.0306] [INSPIRE].

[18] A. Donos and J.P. Gauntlett, Thermoelectric DC conductivities from black hole horizons, JHEP 11 (2014) 081 [arXiv:1406.4742] [INSPIRE].

[19] G. Policastro, D.T. Son and A.O. Starinets, From AdS/CFT correspondence to hydrodynamics, JHEP 09 (2002) 043 [hep-th/0205052] [INSPIRE].

[20] R.A. Davison and B. Goutéraux, Momentum dissipation and effective theories of coherent and incoherent transport, JHEP 01 (2015) 039 [arXiv:1411.1062] [INSPIRE].

[21] S.S. Gubser and F.D. Rocha, Peculiar properties of a charged dilatonic black hole in $A d S_{5}$, Phys. Rev. D 81 (2010) 046001 [arXiv:0911.2898] [inSPIRE].

[22] R.A. Davison, K. Schalm and J. Zaanen, Holographic duality and the resistivity of strange metals, Phys. Rev. B 89 (2014) 245116 [arXiv:1311.2451] [INSPIRE].

[23] S.J. Poletti and D.L. Wiltshire, Global properties of static spherically symmetric charged dilaton spacetimes with a Liouville potential, Phys. Rev. D 50 (1994) 12 [Erratum ibid. D 52 (1995) 3753] [gr-qc/9407021] [INSPIRE].

[24] G.W. Gibbons and D.L. Wiltshire, Black holes in Kaluza-Klein theory, Annals Phys. 167 (1986) 201 [Annals Phys. 176 (1987) 393].

[25] S.J. Poletti, J. Twamley and D.L. Wiltshire, Charged dilaton black holes with a cosmological constant, Phys. Rev. D 51 (1995) 10 [hep-th/9412076] [INSPIRE].

[26] M. Blake, D. Tong and D. Vegh, Holographic lattices give the graviton an effective mass, Phys. Rev. Lett. 112 (2014) 071602 [arXiv:1310.3832] [INSPIRE].

[27] K.-Y. Kim, K.K. Kim, Y. Seo and S.-J. Sin, Coherent/incoherent metal transition in a holographic model, JHEP 12 (2014) 170 [arXiv:1409.8346] [INSPIRE].

[28] P. Kovtun and A. Ritz, Universal conductivity and central charges, Phys. Rev. D 78 (2008) 066009 [arXiv: 0806.0110] [INSPIRE].

[29] A. Donos and J.P. Gauntlett, Novel metals and insulators from holography, JHEP 06 (2014) 007 [arXiv: 1401.5077] [INSPIRE]. 
[30] M. Baggioli and O. Pujolàs, Electron-phonon interactions, metal-insulator transitions and holographic massive gravity, Phys. Rev. Lett. 114 (2015) 251602 [arXiv:1411.1003] [INSPIRE].

[31] A. Amoretti and D. Musso, Universal formulae for thermoelectric transport with magnetic field and disorder, arXiv:1502.02631 [INSPIRE].

[32] N.E. Hussey, Phenomenology of the normal state in-plane transport properties of high- $T_{c}$ cuprates, J. Phys Condes. Matter 20 (2008) 123201. 